\title{
TeaAS: a comprehensive database for alternative splicing in tea plants (Camellia sinensis)
}

Xiaozeng $\mathrm{Mi}^{1+}$, Yi Yue ${ }^{2+}$, Mengsha Tang ${ }^{1}$, Yanlin An ${ }^{1}$, Hui Xie ${ }^{1}$, Dahe Qiao ${ }^{1}$, Zhiyu Ma², Shengrui Liu ${ }^{1}$ and Chaoling Wei $^{1^{*}}$

\begin{abstract}
Alternative splicing (AS) increases the diversity of transcripts and proteins through the selection of different splice sites and plays an important role in the growth, development and stress tolerance of plants. With the release of the reference genome of the tea plant (Camellia sinensis) and the development of transcriptome sequencing, researchers have reported the existence of AS in tea plants. However, there is a lack of a platform, centered on different RNA-seq datasets, that provides comprehensive information on AS.

To facilitate access to information on AS and reveal the molecular function of AS in tea plants, we established the first comprehensive AS database for tea plants (TeaAS, http://www.teaas.cn/index.php). In this study, 3.96 Tb reads from 66 different RNA-seq datasets were collected to identify AS events. TeaAS supports four methods of retrieval of AS information based on gene ID, gene name, annotation (non-redundant/Kyoto encyclopedia of genes and genomes/ gene ontology annotation or chromosomal location) and RNA-seq data. It integrates data pertaining to genome annotation, type of AS event, transcript sequence, and isoforms expression levels from 66 RNA-seq datasets. The AS events resulting from different environmental conditions and that occurring in varied tissue types, and the expression levels of specific transcripts can be clearly identified through this online database. Moreover, it also provides two useful tools, Basic Local Alignment Search Tool and Generic Genome Browser, for sequence alignment and visualization of gene structure.

The features of the TeaAS database make it a comprehensive AS bioinformatics platform for researchers, as well as a reference for studying AS events in woody crops. It could also be helpful for revealing the novel biological functions of AS in gene regulation in tea plants.
\end{abstract}

Keywords: Alternative splicing, Genome and transcriptome, Transcripts, Isoforms, Tea plant

\footnotetext{
*Correspondence: weichl@ahau.edu.cn

${ }^{\dagger}$ Xiaozeng Mi and Yi Yue contributed equally to this work.

1 State Key Laboratory of Tea Plant Biology and Utilization, Anhui

Agricultural University, West 130 Changjiang Road, Hefei, Anhui 230036,

People's Republic of China

Full list of author information is available at the end of the article
}

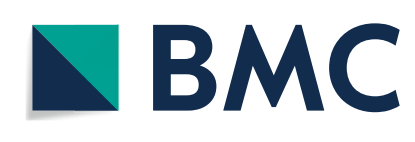

(c) The Author(s) 2021. Open Access This article is licensed under a Creative Commons Attribution 4.0 International License, which permits use, sharing, adaptation, distribution and reproduction in any medium or format, as long as you give appropriate credit to the original author(s) and the source, provide a link to the Creative Commons licence, and indicate if changes were made. The images or other third party material in this article are included in the article's Creative Commons licence, unless indicated otherwise in a credit line to the material. If material is not included in the article's Creative Commons licence and your intended use is not permitted by statutory regulation or exceeds the permitted use, you will need to obtain permission directly from the copyright holder. To view a copy of this licence, visit http://creativecommons.org/licenses/by/4.0/. The Creative Commons Public Domain Dedication waiver (http://creativeco $\mathrm{mmons}$.org/publicdomain/zero/1.0/) applies to the data made available in this article, unless otherwise stated in a credit line to the data. 


\section{Background}

Alternative splicing (AS) refers to pre-mRNA processing events that produce multiple mRNAs from the same gene through variable selection of splice sites. It is an important mode of regulating gene expression and increasing protein diversity [1]. AS can be grouped into four main types: intron retention (IR), alternative 3' splice sites (A3SS), alternative 5' splice sites (A5SS), and exon skipping (ES). AS has been found to be involved in growth and development of cell types, metabolism, and regulates the response to biological and abiotic stress in many plant species [2-4]. For example, the FLOWERING LOCUS M $(F L M)$ is subject to temperature-dependent AS that regulates flowering in Arabidopsis [5]. In addition, the gene involved in the AS of an MYB transcription factor affects anthocyanin biosynthesis in tomato fruits, and mutations in the splice sites cause a complete loss of function in the wild-type protein [6]. Moreover, LlHSFA3B-III, an AS isoform of LlHSFA3B (heat stress transcription factors), was also found to regulate abiotic heat stress in lily plants [7]. In tea plants, an AS-associated analysis of different tissues showed that it could regulate the flavonoid pathway through certain transcription factors and functional genes [8]. In response to cold stress, plants undergo AS to regulate expression of genes involved in sugar metabolism pathways and those coding for antioxidant enzymes [9]. Furthermore, AS isoforms of the CsLOX gene were generated by tea geometrid feeding and Glomerella cingulata infection. Thus, AS plays an important role in plant growth, development and response to stress. AS databases have been established in many other crops, such as cucumber [10]. In addition, a comprehensive AS database of plant communities was established, including that of cotton [11], tomato [12], fruit plants [13], Brachypodium distachyon [14] and other crops. The establishment of these databases enriches the knowledge on gene functions and facilitates access to AS-related information; however, till date, there is no comprehensive database of AS in tea plants (Camellia sinensis).

The tea plant is an important perennial evergreen woody crop of significant economic and cultural importance [15]. Tea leaves are used for making tea beverages, which is one of the three most popular nonalcoholic beverages consumed worldwide. It not only contains polyphenols, catechins, caffeine, and other flavorful compositions, but it also provides health-promoting benefits $[16,17]$. Several databases have been established to facilitate the study of tea plants [18-20], however, these do not include the information associated with AS.

With the release of the reference genome of the tea plant [21-23], researchers have made great progress in the understanding of functional genes and their regulatory mechanisms, which also provides a basis for the identification of AS. Transcriptome sequencing has become an important way to study the biological functions of genes. Recently, transcriptomes in response to different environmental conditions have been analyzed in many plant species $[24,25]$. In tea plants, transcriptome sequencing has been used to understand the expression of genes responsive to low temperature, salinity, drought, and heat stress $[9,26,27]$, and also to reveal the mechanisms of self-incompatibility, secondary metabolism, and development [28-30]. These studies have enriched the understanding of the biological function of genes in tea plants, but they only focused on a single transcript derived from each gene, and did not investigate the function of multiple transcripts arising from AS events. Therefore, the role of AS in these biological processes in tea plants is unclear. Mapping the RNA sequence (obtained by RNA-seq) to the reference genome and assembling the genomes are important steps to identify AS events, which promote the understanding of the biological function of AS. Thus, the objective of this study was to establish a comprehensive AS database of tea plants, the TeaAS, to provide researchers with a public and freely available AS database.

\section{Construction and content}

\section{Data sources}

The genome of the tea plant (Camellia sinensis var. sinensis cv. suchazao) was used as the reference genome [21]. The current release of the tea plant genome and genome annotations were collected from the TPIA platform (http://tpia.teaplant.org/). Sixty-six RNA-seq datasets associated with different environmental conditions or tissue types were downloaded from the SRA database (https://www.ncbi.nlm.nih.gov/sra/). Details of all RNAseq data are provided in Additional file 1: Table S1.

\section{Data processing}

The SRA Toolkit was used to download RNA-seq data from the NCBI Sequence Read Archive (SRA) database. All raw sequencing data were filtered through the Trimmomatic (v0.36) software with the same standard [31]. The adapter sequence was removed by the ILLUMINACLIP parameter with a minimum adapter length of eight. In addition, reads with a continuous 5 bp base average quality below 20 and a length under $36 \mathrm{bp}$ after filtering were filtered out. The remaining paired reads were used for subsequent analyses.

The filtered RNA-seq reads were mapped to the reference genome using Hisat2 (v2.1.0) with default parameters. The generated sequence alignment map (SAM) files were converted to BAM files by Samtools software. Then transcripts were assembled using StringTie software (v.1.3.5) with default settings [32]. Finally, the gene 
transfer format (GTF) files from each sample in the same transcriptome were merged using the StringTie with "merge" parameter.

\section{Identification of AS events}

The online tool AStalavista (http://astalavista.sammeth. net/) was employed to identify the AS events from different transcriptome datasets [33]. Briefly, the merged GTF files were submitted to the website by selecting other organisms, and the other options used default values. Four major types of AS events, including IR, A3SS, A5SS, ES, and multiple splicing events occurring on a single transcript were extracted from the output files and counted, respectively. The reads number of splice sites and differential expression of the AS genes were analyzed by rMATS (v.4.1.1, rnaseq-mats.sourceforge. net/) software [34].

Transcripts per million reads (TPM) were used to quantify the transcript expression levels. The TPM from each sample was calculated using StringTie. Then, the gene ID, transcript ID, and their TPM were extracted to form a dataset of all the transcript expression levels. To avoid the effect of very weakly expressed transcripts on the accuracy of the results, we filtered out transcripts with TPM less than one in all the samples. A gene is considered to have undergone AS only if it has at least two mRNAs are produced from the precursor-mRNA and the TPM of these transcripts is greater than one in at least one sample.

\section{Functional annotation of transcripts}

All amino acid and nucleotide sequences of the fulllength transcripts and AS isoforms were extracted using GTF files generated by Stringtie. NR, GO and KEGG annotation databases were used to compare all transcripts to the annotations of the reference genome. The coding region and amino acid sequence of the newly generated transcripts were predicted using the TransDecoder software (https://github.com/TransDecoder/ TransDecoder, v5.5.0). Only one coding sequence (CDS) per transcript was the output using the "-single_best_ only" parameter and homology search against the UniProt database. To prevent the generation of nonsense codons or short peptides, we used any amino acids greater than or equal to 100 aa for further analysis. The domains information of all amino acids was identified using the Pfamscan software (ftp://ftp.ebi.ac.uk/pub/ databases/Pfam/Tools/).

\section{Utility}

A multistep process was used to construct the TeaAS database (Fig. 1). The TeaAS web interface offers four main functional sections, including Home (search),
Summary, Tools, and Download, to provide information on the AS. In addition, the links section provides users with links to the genome and other databases of tea species, Users can provide feedbacks through the "Contacting Us" section.

\section{General functions}

The home page contains the search interface (Fig. 2). The users can search the database using the gene ID (e.g., CSS0013065.1), gene name (e.g., bZIP transcription factor), gene annotation [non-redundant (NR), Kyoto Encyclopedia of Genes and Genomes (KEGG), gene ontology (GO) annotation, and chromosomal location], and RNAseq datasets (e.g., drought stress). Search results are represented on the results page in two ways. First, a list of genes and associated information would be presented if the user conducted the search for all the above-mentioned annotations and for varying environmental conditions (Fig. 3a). Each gene in the list can be double-clicked to determine the environmental condition upon which an AS event has occurred. The transcript and protein products of the AS event can be visualized after a gene and treatment are selected. Second, the environmental conditions and genetic information are rendered when the user searches with gene IDs and gene families (Fig. 3b). Subsequently, the details of the AS event can be browsed by clicking on one environmental condition.

\section{Database overview}

Data and statistical information can be found on the summary page (Additional file 2: Figure S1). This page provides: (I) the transcriptome information, which includes an introduction to the transcriptome, the NCBI accession number, information for each treatment sample, and corresponding publications; (II) statistics of the four major types of AS events and the number of AS genes (Additional file 3: Table S2); (III) the percentage of AS genes among all annotated genes; (IV) the distribution of AS genes on 15 chromosomes; (V) annotation information of all AS genes; and (VI) percent spliced in (PSI) between treatments and the number of reads at splice sites (Additional file 4: Table S3).

The results showed that a total of 131,924 AS events were identified in 11,320 genes in the transcriptome of PRJNA576575, whereas only 9,152 AS events were identified in a self-incompatibility transcriptome (PRJNA355226). This may be due to the differences in treatment conditions of the tea plants and the size of the sequencing data. A total of 28,648 genes underwent AS, which accounts for $56.7 \%$ of the total number of annotated genes $(50,525)$. Moreover, only $0.56 \%$ of the genes that underwent AS were common in all the transcriptome datasets. 


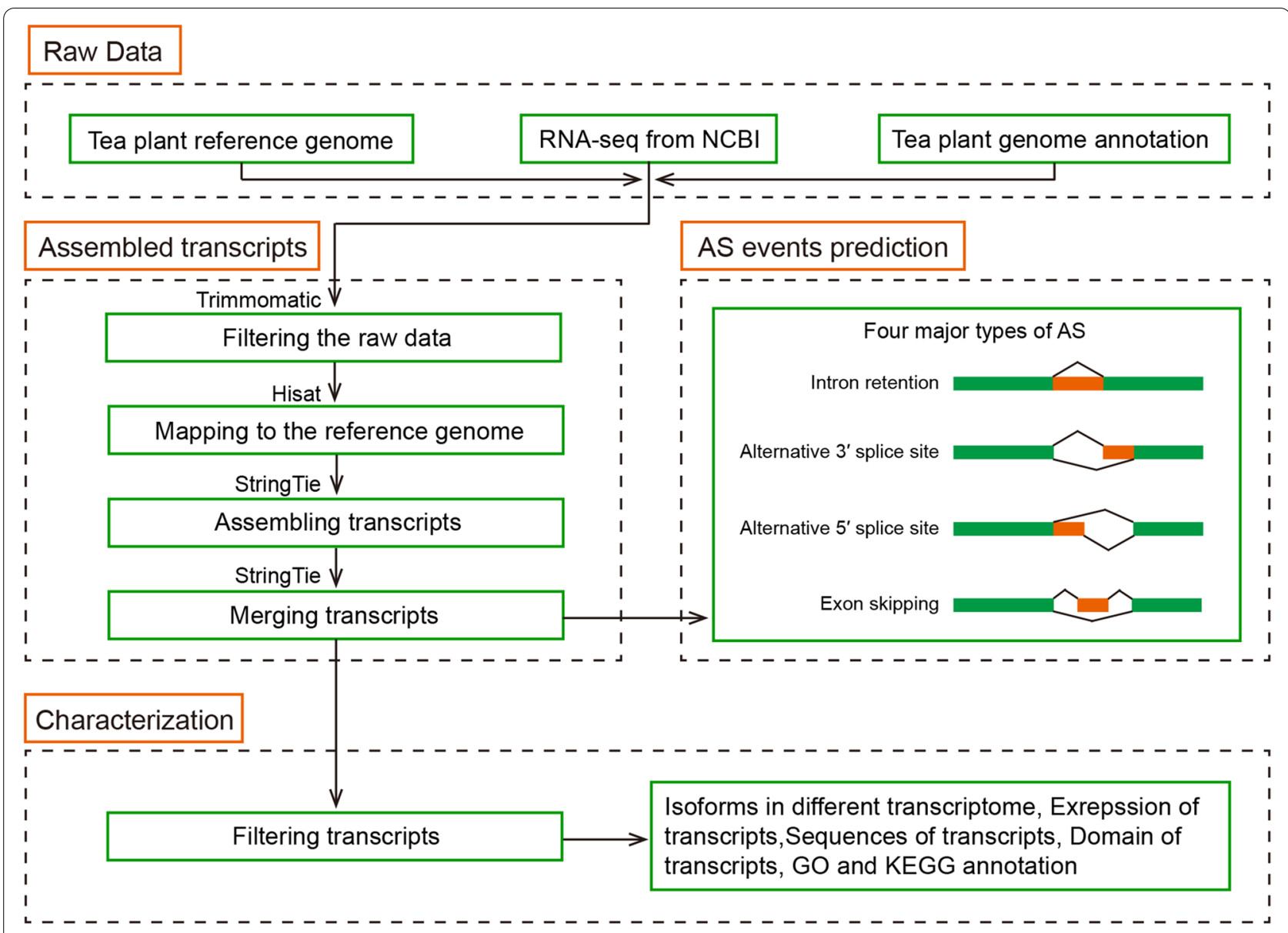

Fig. 1 Workflow of the construction of the TeaAS database

\begin{tabular}{|l|l|}
\hline Search by annotation & Search \\
\hline GO:00302426 & Search \\
\hline Search by gene ID & SS0011081.1 \\
\hline Search by gene name & Search \\
\hline WRKY transcription factor & Search \\
\hline Salinity stress and drought stress
\end{tabular}

Fig. 2 Search tools of TeaAS 


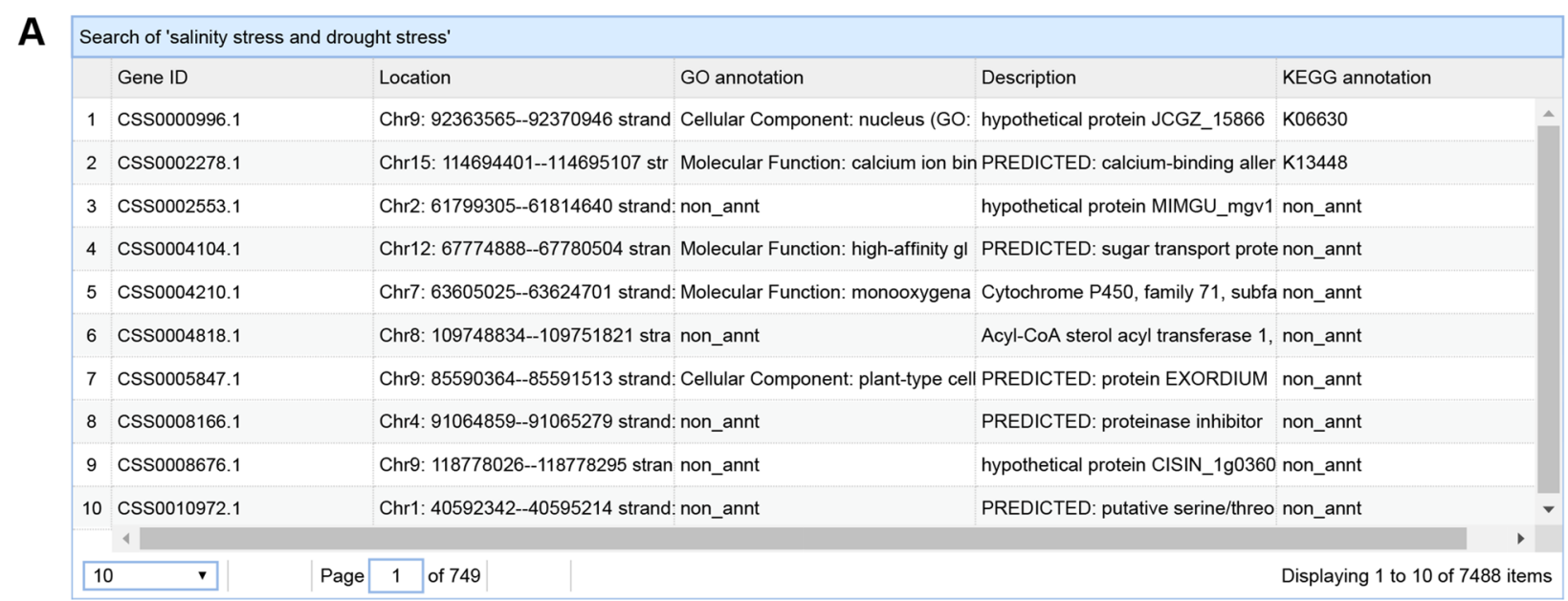

\section{B Gene result 1}

$\begin{array}{lll}\text { Gene ID: } & \text { Description: } & \text { Location: } \\ \text { CSS0043153.1 } & \text { chitinase } & \text { Chr1: 168777315--168779426 strand:+ }\end{array}$

GO annotation: Molecular Function: chitinase activity (GO:0004568);Biological Process: carbohydrate metabolic process (GO:0005975);Biological Process: chitin catabolic process (GO:0006032); Molecular Function: chitin binding (GO:0008061);Biological Process: cell wall mac

\section{Click one RNA-seq data to view AS information}

different nitrogen treatment $\square$

Fig. 3 Search results by gene ID, gene name and RNA-seq datasets. a The list of genetic information based on gene annotation and RNA-seq datasets; and $\mathbf{b}$ results page upon searching by the gene ID and gene name

\section{Other functions}

TeaAS mainly provides users with two effective tools, Basic Local Alignment Search Tool (BLAST) [35] and Generic Genome Browser (GBrowse). BLAST can be used to find homologous sequences in the tea plant. Users can compare the query sequences with any of the assembled transcripts from the 66 transcriptome datasets. The results could then be analyzed for the occurrence of AS events. GBrowse was used to visualize the gene structure of tea plants, including the transcripts from 66 transcriptome datasets.

The download page allows users to download all the data for free. The assembled GTF files, AS event identification files, and all transcription expression files from 66 transcriptome datasets can be downloaded from this page. Moreover, the results of the search can also be downloaded directly. In addition, TeaAS provides a link to the tea plant genome website and to the other tea plant databases on the link page.

\section{Usage cases}

Case study 1, To search for AS events in a gene: The users can directly use the gene name and gene ID to search whether the gene is capable of undergoing AS. At the same time, users can also submit a sequence for confirming the gene ID by the BLAST tool. For example, a search with the ID CSS0005154.1 yields the result that the gene 
encodes a NAC transcription factor and undergoes AS events under multiple treatment conditions, such as salt stress, exposure to sucrose, and cold acclimation. In addition, users can identify genes that undergo AS a specific treatment. For instance, many disease-resistant genes and transcription factors undergo AS under conditions of gray blight disease.

Case study 2, To obtain details associated with AS genes: For example, retrieval based on the gene ID CSS0011081.1 yields the WRKY gene, which is located on chromosome 14, encodes a transcription factor,and has no GO and KEGG annotations. Furthermore, the conditions under which AS events have occurred can also be identified, such as anthracnose diseases, in different tissue types, and upon shade treatments. After clicking on "different tissues 2," we determined that this gene undergoes AS and yields two transcripts, CSS0011081.1 and MSTRG.14319.1 (Fig. 4a). The structure of these two transcripts is shown in Fig. 4b, where the second exon of the AS isoform is missing. The RNA sequence, coding sequence (CDS), and amino acid sequences of the transcripts can be viewed directly from the sequence options (Fig. 4c). The two transcripts were found to have the highest expression levels in the flowers through the data of expression levels (Fig. 4d). Although a $30 \mathrm{bp}$ nucleic acid sequence was lacking in the AS isoform, this sequence was not present in the WRKY domain (Fig. 4e).

\section{Discussion}

AS is a gene regulatory mechanism that has been shown to play an important role in plant growth and development and in responses to biotic and abiotic stresses $[1,2,36]$. In tea plants, AS is involved in gene expression associated with metabolism [37], response to low temperature [9], and drought stress [38]. However, there is a lack of data available for researchers regarding AS in tea plants. In this study, we have established, for the first time, a comprehensive AS database for tea plants. The datasets included in our database can be used as a reference for AS, and the abundant transcriptome data can provide basic information on gene function.

AS databases have been reported in other plants. For example, an AS database for cucumbers, named CuAS, provides detailed information on AS events and transcripts [10]. This database provides information on the AS isoform sequences, domains, and expression levels. In addition, a large number of tissue-specific AS events have been identified in CuAS. However, sequencing data from only 17 different tissues of two cucumber species were included in this database, which is far from what is sufficient to fully understand the AS in cucumber plants. Another AS database was established in tomatoes [12], wherein 300,665 expressed sequence tag sequences and 27 transcriptome datasets were used to identify AS. Compared to the cucumber database, the tomato database represented a variety of tissue types, however, it only provided a search page with limited AS information. To enrich the content of the database established in this study, we collected 66 transcriptome datasets obtained upon exposure to different environmental conditions for a total of $3.96 \mathrm{~Tb}$ sequencing reads, making the identification of AS in tea plants accurate and comprehensive. Additionally, more information about full-length transcripts and AS isoforms is presented on our website, and useful tools for analysis are provided. Thus, TeaAS can be used as a reference for the AS in tea plants. With the publication of a high-quality tea plant genome sequence, the Tea Plant Information Archive (TPIA) was established [18], which contains information on the tea plant genome, transcriptome, and metabolome. It provides a powerful tool for analyzing genomic data and elucidating biological mechanisms of growth and development in tea plants. The database of simple sequence repeat (SSR) markers [19] and co-expression networks [20] have been established in tea plants, providing a basis for the study of functional genes and breeding in tea plants. However, a database related to AS has not been reported yet. The establishment of TeaAS would provide an important tool for studying gene regulation in relation to AS in tea plants.

TeaAS provides a comprehensive information on AS by mapping RNA-seq data to the reference genome. In recent years, with the development of sequencing technology, numerous transcriptome datasets have been sequenced. For instance, transcriptome sequencing has revealed that several genes involved in sugar metabolism, proline metabolism, induction of $\mathrm{CBF}$ (C-repeat binding factor) expression, and the cold-responsive (ICE-CBF - COR) pathway play key roles in cold acclimation in tea plants [39]. Using the same data for AS analysis, researchers found that AS could also affect the sugar metabolism pathway. Furthermore, AS may respond to low temperatures via oxidoreductase and transcription factors (e.g. bHLH, WRKY) [9]. In addition, there have been several reports identifying genes related to anthocyanin synthesis in tea plants [40, 41]. In fact, the synthesis of anthocyanin was not only regulated by the full-length transcripts, but also by AS isoforms of genes such as anthocyanidin reductase (ANR) and anthocyanidin synthase (ANS) [37]. These results indicated that AS plays an important role in regulating metabolism and stress responses of tea plants. To facilitate the acquisition of AS information, TeaAS provides researchers with a simple search interface. 
A

AS type

\section{Type: \\ Exon Skipping}

C

\section{MSTRG.14319.1 FA Sequcece}

ACTTGACCAAAACAAAAAAGACACGGAAAATCTTTGTTTGTGGGTGC AATGGAATCAAGAATGGATTATACAAGTCTCAACCTCAACCTCAACA TTAATCCTCAAGACAACATTGATGAAACTACAGAAAGTGTTTTGATG GAAAAGTTGAACAAGATGAGTAGTGAGAACAAGAAGCTAACAGAGAT GCTGTTTTTGATTTGTGAAAATTACAATGCTTTGCAAGGCCACTTAG TCGACTCATCCHMTGACTCTCAAATAATAACCOCCAAACTACC AAATCAAAGAAGAGACAGTGTGAAGGTGGTGAGAACTCTGGTGGAGC TAACAATGAGAGCAGCTCATGTAGTGATGGAGGTTCATGTAAGAGGC CAAGGGAAATCAAGACTAGTGTTTCAAGGGTTTGTGTGAAGGTTGAT TCATCTGATACAAGACTAATAGTAAAGGATGGATATCAATGGAGAAA ATATGGACAAAAGGTCACTAGAGATAACCCATCTCCTAGAGCTTACT ACAAGTCCTCTTATGCACCAAGCTGCCCAGTTAAAAGAACGTCCAA AGAAGTCTTCAAGATCCCTCAATAGTGGTAGCAACCTATGAAGGAGA CCACAACCACAGCCACCCATGTCAAGCTGAGGTGGCTCTGGGCTTGA ACCAGTTTGTCTCCCCTACCATGATCACTTCAGAAAGCCCTACTACT ACTCCAAATCCAAATCCAATGATTATTGATTCCACACAACTAGGACT GTTTGGTAATGCCAACAAGTCTATTCCAGAGATTGATCTTCCTGCAT TTCAAAATCTTTTGGTTGAAAAAATGGCTAATTCTCTAACAAAAAAT CACACTTTCACTACACCTCTTCCACCACCCATTTCAACCAAGATTCT AGACCTAGAGGGTTGGGAAAACAGAAGTGTCTAATTTTGAGTACAAT TTTCTTTTTCTTTTTTTCTTTTAATTTTTAAATTTCATGTAAGAGTA TTGTTTCTGAAGATATGAGTAGGGTCACTATCAACTGAAGTGAATGT AATTTTATGTAGAAATGCAAAAAATTATATTGTCTTCT

\section{D}

\section{CSS0011081.1 Expression Graph}

Transcripts per million (TPM)

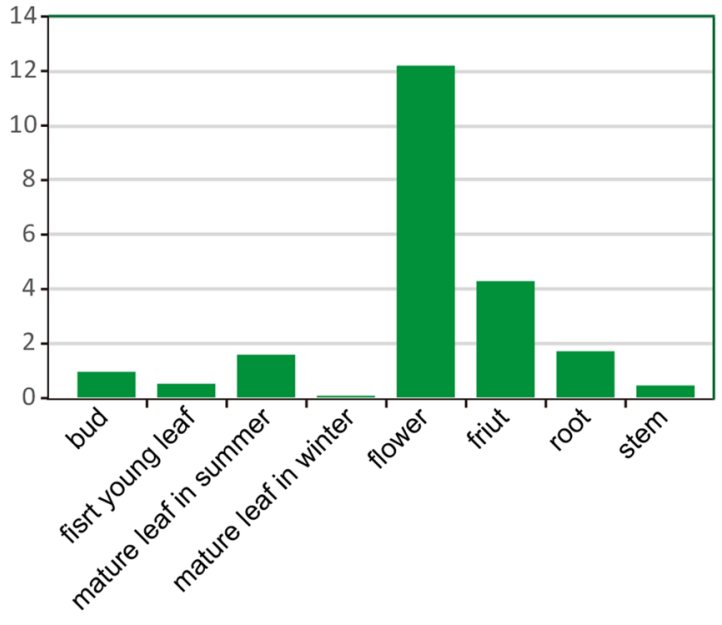

B

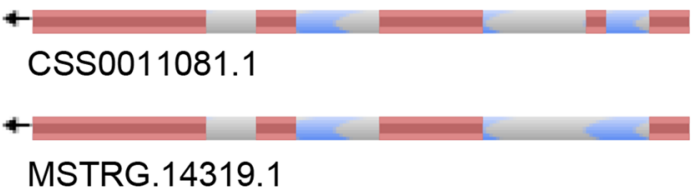

\section{MSTRG.14319.1 CDS Sequcece:}

ATGGAATCAAGAATGGATTATACAAGTCTCAACCTCAACC TCAACATTAATCCTCAAGACAACATTGATGAAACTACAGA AAGTGTTTTGATGGAAAAGTTGAACAAGATGAGTAGTGAG AACAAGAAGCTAACAGAGATGCTGTTTTTGATTTGTGAAA ATTACAATGCTTTGCAAGGCCACTTAGTGGACTTGATGCA AAAGAACTCTCAAMTAATAMCCACCAMACTACCHATCA CTAACAATGAGAGCAGCTCATGTAGTGATGGAGGTTCATG TAAGAGGCCAAGGGAAATCAAGACTAGTGTTTCAAGGGTT TGTGTGAAGGTTGATTCATCTGATACAAGACTAATAGTAA AGGATGGATATCAATGGAGAAAATATGGACAAAAGGTCAC TAGAGATAACCCATCTCCTAGAGCTTACTACAAGTGCTCT TATCCACCHACTCCCCACTTAAAAGACCTCCHAGA TATGCACCAAGCTGCCCAGTTABAAGAGGTGCAAAGAA AGACCACAACCACAGCCACCCATGTCAAGCTGAGGTGGCT CTGGGCTTGAACCAGTTTGTCTCCCCTACCATGATCACTT CAGAAAGCCCTACTACTACTCCAAATCCAAATCCAATGAT TATTGATTCCACACAACTAGGACTGTTTGGTAATGCCAAC AAGTCTATTCCAGAGATTGATCTTCCTGCATTTCAAAATC TTTTGGTTGAAAAAATGGCTAATTCTCTAACAAAAAATCA CAGTTTCACTACAGCTCTTGCAGCAGCCATTTCAACCAAG ATTCTAGACCTAGAGGGTTGGGAAAACAGAAGTGTCTAA

E

\section{MSTRG.14319.1 Expression Graph}

Transcripts per million (TPM)

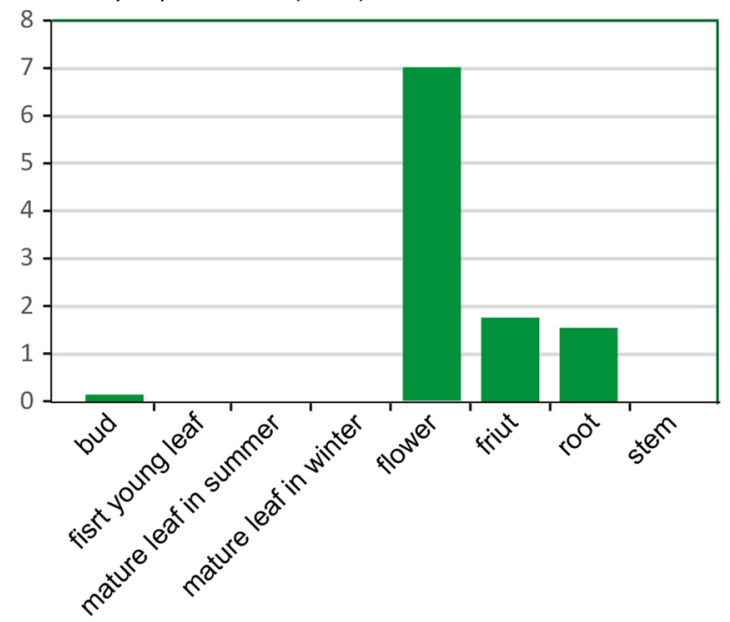

\begin{tabular}{|c|c|c|c|c|c|c|c|c|}
\hline \multicolumn{9}{|c|}{ Pfam Data } \\
\hline & Seq id & \multicolumn{2}{|c|}{ alignment start } & alignment end & envelope start & envelope end & $\mathrm{hmm}$ acc & hmm name \\
\hline 1 & CSS0011081.1 & \multicolumn{2}{|r|}{154} & 211 & 154 & 211 & PF03106.15 & WRKY \\
\hline \multirow[t]{2}{*}{2} & MSTRG.14319.1 & \multicolumn{2}{|r|}{134} & 191 & 134 & 192 & PF03106.15 & WRKY \\
\hline & \multicolumn{8}{|c|}{4} \\
\hline 10 & $\cdot|1<<| F$ & Page [ & 1 of $1 \mid>$ & $\triangle \circlearrowright$ & & & \multicolumn{2}{|c|}{ Displaying 1 to 2 of 2 items } \\
\hline
\end{tabular}

Fig. 4 Alternative splicing information of TeaAS. a AS type and transcripts ID; $\mathbf{b}$ gene structure of full-length transcripts and AS isoforms; $\mathbf{c}$ nucleotide and amino acid sequences of transcripts; $\mathbf{d}$ expression levels of transcripts in each sample, and e domain information of transcripts 


\section{Conclusions}

In summary, TeaAS is a platform that provides comprehensive information on full-length transcripts and their AS isoforms in tea plants. The establishment of TeaAS provides a reference for AS and would be helpful in potential role of AS in gene regulation via comparative analyses in tea plants.

\section{Abbreviations}

TeaAS: A database for alternative splicing in tea plant; AS: Alternative splicing; IR: Intron retention; A3SS: Alternative 3' splice sites; A5SS: Alternative 5' splice sites; ES: Exon skipping; TPM: Transcripts per million reads; NR: Non-redundant protein; GO: Gene ontology; KEGG: Kyoto Encyclopedia of Genes and Genomes; CDS: Coding sequence.

\section{Supplementary Information}

The online version contains supplementary material available at https://doi. org/10.1186/s12870-021-03065-8.

Additional file 1: Table S1. Details of 66 RNA-seq dataset.

Additional file 2: Figure S1. Statistics of 66 RNA-seq datasets and AS events.

Additional file 3. Table S2. Statistics of four major AS events in 66 RNAseq datasets.

Additional file 4: Table S3. Information of differentially expressed AS by rMATS analysis.

\section{Acknowledgements}

Not applicable.

\section{Authors' contributions}

CLW designed this project. XZM, YY, MST, YLA, HX, DHQ, ZYM and SRL collected and analyzed the transcriptome sequencing data. $X Z M, Y Y$, and $Z Y M$ designed the web interface and maintained the server. XZM wrote this article. All authors have read and approved the final manuscript.

\section{Funding}

This work was supported by the National Natural Science Foundation of China (U20A2045), the National Key Research and Development Program of China (2019YFD1001601), Project of Major Science and Technology in Anhui Province (202003a06020021) and the Base of Introducing Talents for Tea plant Biology and Quality Chemistry (D20026), the special funds for the tea germplasm resource garden (2060502 and 201834040003), the Open Fund of State Key Laboratory of Tea Plant Biology and Utilization, SKLTOF20150103, and the National Natural Science Foundation of China (31800585).

\section{Availability of data and materials}

The genomic data of the tea plant (Camellia sinensis var. sinensis cv. suchazao) are available at TPIA platform (http://tpia.teaplant.org/download.html). The RNA-seq datasets supporting the results of this article are available at the SRA database of $\mathrm{NCBI}$ (https://www.ncbi.nlm.nih.gov/), and all relevant accession numbers and details are provided in TeaAS (http://www.teaas.cn/static/html/ Browse.html).

\section{Declarations}

Ethics approval and consent to participate Not applicable.

\section{Consent for publication}

Not applicable.

\section{Competing interests}

The authors declare that they have no conflict of interest.

\section{Author details}

${ }^{1}$ State Key Laboratory of Tea Plant Biology and Utilization, Anhui Agricultural University, West 130 Changjiang Road, Hefei, Anhui 230036, People's Republic of China. ${ }^{2}$ School of Information and Computer, Anhui Agricultural University, West 130 Changjiang Road, Hefei, Anhui 230036, People's Republic of China.

Received: 16 February 2021 Accepted: 25 May 2021

Published online: 21 June 2021

\section{References}

1. Yang SM, Tang F, Zhu HY. Alternative Splicing in Plant Immunity. Int J Mol Sci. 2014;15(6):10424-45.

2. Laloum T, Martin G, Duque P. Alternative Splicing Control of Abiotic Stress Responses. Trends Plant Sci. 2018;23(2):140-50.

3. Zhang H, Mao R, Wang YZ, Zhang L, Wang CY, Lv SK, Liu XL, Wang YJ, Ji $W Q$. Transcriptome-wide alternative splicing modulation during plantpathogen interactions in wheat. Plant Sci. 2019;288:110160.

4. Dong CL, He F, Berkowitz O, Liu JX, Cao PF, Tang M, Shi HC, Wang WJ, Li $\mathrm{QL}$, Shen ZG, et al. Alternative Splicing Plays a Critical Role in Maintaining Mineral Nutrient Homeostasis in Rice (Oryza sativa). Plant Cell. 2018;30(10):2267-85

5. Pose D, Verhage L, Ott F, Yant L, Mathieu J, Angenent GC, Immink RGH, Schmid M. Temperature-dependent regulation of flowering by antagonistic FLM variants. Nature. 2013:503(7476):414.

6. Colanero S, Tagliani A, Perata P, Gonzali S. Alternative Splicing in the Anthocyanin Fruit Gene Encoding an R2R3 MYB Transcription Factor Affects Anthocyanin Biosynthesis in Tomato Fruits. Plant Commun. 2020;1(1):100006.

7. Wu Z, Liang JH, Wang CP, Ding LP, Zhao X, Cao X, Xu SJ, Teng NJ, Yi MF. Alternative Splicing Provides a Mechanism to Regulate LIHSFA3 Function in Response to Heat Stress in Lily. Plant Physiol. 2019;181(4):1651-67.

8. Zhu JY, Wang XW, Xu QS, Zhao SQ, Tai YL, Wei CL. Global dissection of alternative splicing uncovers transcriptional diversity in tissues and associates with the flavonoid pathway in tea plant (Camellia sinensis). Bmc Plant Biol. 2018;18(1):266.

9. Li YY, Mi XZ, Zhao SQ, Zhu JY, Guo R, Xia XB, Liu L, Liu SR, Wei CL. Comprehensive profiling of alternative splicing landscape during cold acclimation in tea plant. Bmc Genomics. 2020;21(1):65.

10. Sun Y, Zhang QB, Liu B, Lin K, Zhang ZH, Pang EL. CuAS: a database of annotated transcripts generated by alternative splicing in cucumbers. Bmc Plant Biol. 2020;20(1):119.

11. Min XJ. A Survey of Alternative Splicing in Allotetraploid Cotton (Gossypium hirsutum L.). Computational Molecular Biology. 8(1):1-13.

12. Clark S, Yu F, Gu LF, Min XJ. Expanding Alternative splicing identification by integrating multiple sources of transcription data in tomato. Front Plant Sci. 2019;10(689).

13. Sablok G, Powell B, Braessler J, Yu F, Min XJJCPB. Comparative landscape of alternative splicing in fruit plants. Current Plant Biology. 2017;9(C):29-36.

14. Walters B, Lum G, Sablok G, Min XJ. Genome-Wide Landscape of Alternative Splicing Events in Brachypodium distachyon. DNA Res. 2013;20(2):163-71.

15. Xia EH, Tong W, Wu Q, Wei S, Zhao J, Zhang ZZ, Wei CL, Wan XC. Tea plant genomics: achievements, challenges and perspectives. Hortic ResEngland. 2020;7(1):7.

16. Zhang L, Ho CT, Zhou J, Santos JS, Armstrong L, Granato D. Chemistry and Biological Activities of Processed Camellia sinensis Teas: A Comprehensive Review. Compr Rev Food Sci F. 2019;18(5):1474-95.

17. Zhou J, Ho CT, Long PP, Meng QL, Zhang L, Wan XC. Preventive Efficiency of Green Tea and Its Components on Nonalcoholic Fatty Liver Disease. J Agr Food Chem. 2019;67(19):5306-17.

18. Xia EH, Li FD, Tong W, Li PH, Wu Q, Zhao HJ, Ge RH, Li RP, Li YY, Zhang ZZ, et al. Tea Plant Information Archive: a comprehensive 
genomics and bioinformatics platform for tea plant. Plant Biotechnol J. 2019;17(10):1938-53.

19. Dubey H, Rawal HC, Rohilla M, Lama U, Kumar PM, Bandyopadhyay T, Gogoi M, Singh NK, Mondal TK. TeaMiD: a comprehensive database of simple sequence repeat markers of tea. Database-Oxford. 2020.

20. Zhang R, Ma Y, Hu XY, Chen Y, He XL, Wang P, Chen Q, Ho CT, Wan XC, Zhang $\mathrm{YH}$, et al. TeaCoN. a database of gene co-expression network for tea plant (Camellia sinensis). Bmc Genomics. 2020;21 (1):461.

21. Xia EH, Tong W, Hou Y, An YL, Chen LB, Wu Q, Liu YL, Yu J, Li FD, Li RP, et al. The Reference Genome of Tea Plant and Resequencing of 81 Diverse Accessions Provide Insights into Its Genome Evolution and Adaptation. Mol Plant. 2020;13(7):1013-26.

22. Chen JD, Zheng C, Ma JQ, Jiang CK, Ercisli S, Yao MZ, Chen L. The chromosome-scale genome reveals the evolution and diversification after the recent tetraploidization event in tea plant. Hortic Res-England. 2020;7(1):63.

23. Wang $X C$, Feng $H$, Chang $Y X, M a C L$, Wang $L Y$, Hao XY, Li AL, Cheng H, Wang $L$, Cui P, et al. Population sequencing enhances understanding of tea plant evolution. Nat Commun. 2020;11(1):4447.

24. Cheng ZH, Yu X, Li SX, Wu Q. Genome-wide transcriptome analysis and identification of benzothiadiazole-induced genes and pathways potentially associated with defense response in banana. Bmc Genomics. 2018;19(1):454

25. Wang H, Chang XX, Lin J, Chang YH, Chen JC, Reid MS, Jiang CZ. Transcriptome profiling reveals regulatory mechanisms underlying corolla senescence in petunia. Hortic Res-England. 2018;5(1):16.

26. Wan SQ, Wang WD, Zhou TS, Zhang YH, Chen JF, Xiao B, Yang YJ, Yu YB. Transcriptomic analysis reveals the molecular mechanisms of Camellia sinensis in response to salt stress. Plant Growth Regul. 2018;84(3):481-92.

27. Jiazhi, Shen, Dayan, Zhang, Lin, Zhou, Xuzhou, Jieren, Liao, physiology DJT. Transcriptomic and metabolomic profiling of Camellia sinensis L. Cv. 'Suchazao' exposed to temperature stresses reveals modification in protein synthesis and photosynthetic and anthocyanin biosynthetic pathways. Tree physiology, 2019;39(9):1583-99.

28. Zhang CC, Wang LY, Wei K, Wu LY, Li HL, Zhang F, Cheng H, Ni DJ. Transcriptome analysis reveals self-incompatibility in the tea plant (Camellia sinensis) might be under gametophytic control. Bmc Genomics. 2016;17(1):359.

29. Xu QS, He YX, Yan XM, Zhao SQ, Zhu JY, Wei CL. Unraveling a crosstalk regulatory network of temporal aroma accumulation in tea plant (Camellia sinensis) leaves by integration of metabolomics and transcriptomics. Environ Exp Bot. 2018;149:81-94.

30. Liu F, Wang Y, Ding Z, Zhao L, Xiao J, Wang L, Ding S. Transcriptomic analysis of flower development in tea (Camellia sinensis (L.)). Gene. 2017;631:39-51.
31. Bolger A, Lohse M, Usadel B. Trimmomatic: a flexible trimmer for Illumina sequence data. Bioinformatics. 2014;30(15):2114-20.

32. Pertea M, Kim D, Pertea GM, Leek JT, Salzberg SL. Transcript-level expression analysis of RNA-seq experiments with HISAT StringTie and Ballgown. Nat Protoc. 2016;11(9):1650-67.

33. Foissac S, Sammeth M. ASTALAVISTA: dynamic and flexible analysis of alternative splicing events in custom gene datasets. Nucleic Acids Res. 2007;35:W297-9.

34. Shen SH, Park JW, Lu ZX, Lin L, Henry MD, Wu YN, Zhou Q, Xing Y. rMATS: Robust and flexible detection of differential alternative splicing from replicate RNA-Seq data. P Natl Acad Sci USA. 2014;111(51):E5593-601.

35. Priyam A, Woodcroft BJ, Rai V, Munagala A, Wurm Y. Sequenceserver: a modern graphical user interface for custom BLAST databases. 2015.

36. Li YP, Dai C, Hu CG, Liu ZC, Kang CY. Global identification of alternative splicing via comparative analysis of SMRT- and Illumina-based RNA-seq in strawberry. Plant J. 2017;90(1):164-76.

37. Chen L, Shi X, Nian B, Duan S, Jiang B, Wang X, Lv C, Zhang G, Ma Y, Zhao M. Alternative Splicing Regulation of Anthocyanin Biosynthesis in Camellia sinensis var. assamica Unveiled by PacBio Iso-Seq. G3 (Bethesda). 2020;10(8):2713-23.

38. Ding YQ, Wang Y, Qiu C, Qian WJ, Xie H, Ding ZT. Alternative splicing in tea plants was extensively triggered by drought, heat and their combined stresses. Peerj. 2020;8(2):e8258.

39. Ban $Q Y$, Wang XW, Pan C, Wang YW, Kong L, Jiang HG, Xu YQ, Wang WZ, Pan YT, Li YY, et al. Comparative analysis of the response and gene regulation in cold resistant and susceptible tea plants. PLoS One. 2017;12(12):e0188514.

40. Li J, Lv XJ, Wang LX, Qiu ZM, Song XM, Lin JK, Chen W. Transcriptome analysis reveals the accumulation mechanism of anthocyanins in 'Zijuan' tea (Camellia sinensis var. asssamica (Masters) kitamura) leaves. Plant Growth Regul. 2017:81(1):51-61.

41. Wang L, Pan D, Meng L, Yakubu A, Li J, Lin J, Chen S, Chen WJIJoMS, Regulation of Anthocyanin Biosynthesis in Purple Leaves of Zijuan Tea (Camellia sinensis var. kitamura). 2017.

\section{Publisher's Note}

Springer Nature remains neutral with regard to jurisdictional claims in published maps and institutional affiliations.
Ready to submit your research? Choose BMC and benefit from:

- fast, convenient online submission

- thorough peer review by experienced researchers in your field

- rapid publication on acceptance

- support for research data, including large and complex data types

- gold Open Access which fosters wider collaboration and increased citations

- maximum visibility for your research: over $100 \mathrm{M}$ website views per year

At BMC, research is always in progress.

Learn more biomedcentral.com/submissions 